\title{
Wave Climate Scatter Performance of a Cycloidal Wave Energy Converter
}

\author{
S. G. Siegel \\ Atargis Energy Corporation, 1111 Lavender Way, Pueblo, Colorado, 81001, USA.
}

\begin{abstract}
A lift based Cycloidal Wave Energy Converter (WEC) was investigated using potential flow numerical simulations in combination with viscous loss estimates based on published hydrofoil data. This type of wave energy converter consists of a shaft with one or more hydrofoils attached eccentrically at a radius. The main shaft is aligned parallel to the wave crests and submerged at a fixed depth. The operation of the WEC as a wave-to-shaft energy converter interacting with straight crested waves was estimated for an actual ocean wave climate. The climate chosen was the climate recorded by a buoy off the north-east shore of Oahu/Hawaii, which was a typical moderate wave climate featuring an average annual wave power $P_{W}=17 \mathrm{kWh} / \mathrm{m}$ of wave crest. The impact of the design variables radius, chord, span and maximum generator power on the average annual shaft energy yield, capacity factor and power production time fraction were explored. In the selected wave climate, a radius $R=5 \mathrm{~m}$, chord $C=5 \mathrm{~m}$ and span of $S=60 \mathrm{~m}$ along with a maximum generator power of $P_{G}=1.25 \mathrm{MW}$ were found to be optimal in terms of annual shaft energy yield. At the design point, the CycWEC achieved a wave-to-shaft power efficiency of $70 \%$. In the annual average, $40 \%$ of the incoming wave energy was converted to shaft energy, and a capacity factor of $42 \%$ was achieved. These numbers exceeded the typical performance of competing renewables like wind power, and demonstrated that the WEC was able to convert wave energy to shaft energy efficiently for a range of wave periods and wave heights as encountered in a typical wave climate.
\end{abstract}

Keywords: Wave Energy Conversion, Cycloidal Turbine, Deep Ocean Wave, Cycloidal Wave Energy Converter, Cost of Energy, Wave Scatter Diagram

\section{Introduction}

There is good consensus in published literature that ocean waves are an attractive renewable energy source, as is for example detailed in Boyle [1]. The attractiveness of this resource is due to a large global availability, high energy density as well as consistent availability. With a large portion of the world's population located near the ocean shores, the resource wave energy is well co-located with human consumption of electricity. This enables wave energy to provide power to human settlements without having to add major transmission lines with the related losses and cost of installation. Despite the attractiveness of the resource, at present there are no wave energy installations in commercial operation. Reasons cited for this situation are the high cost and low amounts of power production of present day wave energy converter designs that have reached commercial maturity.

This lack of successful commercial devices is in stark contrast to a plethora of wave energy converter designs, which is best appreciated by a review of the number of patent applications related to the subject. Thus, a comprehensive review of all relevant wave energy converter designs would be prohibitively long, the reader is instead referred to comprehensive reviews published by McCormick [2], Mei [3] or, most recently, Cruz [4]. The following discussion will instead focus only on select devices most pertinent to the current work, where the main feature of the Cycloidal Wave Energy converter is the use of hydrofoils for interaction with the wave induced flow field.

Relatively few publications investigate the interaction of hydrofoils with surface waves for the purpose of wave energy conversion. Wu [5] analyzed the interaction of an oscillating hydrofoil with waves. He identified by means of variational calculus optimal oscillating parameters and reported that a net energy gain could be obtained. Grue et al. [6] explored the possibility of using energy recovered from waves using an oscillating hydrofoil as a means of propulsion for a vessel. They investigated the possible parameter space in detail using potential flow simulations, 
and found that, in particular for waves traveling in the opposite direction of the vessel, a large amount of propulsion energy can be extracted from the waves. This concept was further pursued by Isshiki [7], Isshiki and Murakami [8], and Isshiki et al. [9], resulting in a vessel crossing the Pacific ocean from Japan to the island of Hawaii entirely powered by wave energy (Terao [10]).

A single rotating hydrofoil as a means to convert wave to shaft power was first investigated by Hermans et al.[11] both numerically and experimentally. While Marburg [12] reported very low wave energy conversion efficiencies (on the order of a few percent) in these experimental investigations, Siegel et al.[13] were able to show in simulations that with improved sizing of the WEC as well as by using synchronization of the rotation of the foil with the incoming harmonic wave, wave termination with better than $99 \%$ inviscid efficiency was possible. These numerical findings were confirmed by 1:300 scale experiments in 2011, as reported by Siegel et al.[14] where invisicid conversion efficiencies of greater than $95 \%$ were achieved.

The WEC investigations were subsequently advanced by investigation irregular wave cancellation modeled by a Bretschneider distribution. This was achieved in simulations reported by Jeans et al.[15], as well as experimentally validated by Siegel et al. [16] in a small 2D wave flume. In a follup-up investigation to Jeans et al.[15] which was reported by Fagley et al. [17], an improved controller achieved cancellation of irregular waves with efficiencies almost identical to the earlier harmonic wave cancellation results reported by Siegel et al.[13]. These findings justify the use of harmonic simulations, which are numerically less expensive, for parameter studies of the type reported here. The most recent investigations advanced the experiments to a scale of 1:10 in a three dimensional wave basin, see Fagley et al.[18]. At this scale, successful electricity production was achieved and quantitatively measured for the first time. In addition, the three dimensional wave radiation pattern of a CycWEC was experimentally observed and measured. These observations triggered the development of a numerical radiation model to explore and quantify the impact of finite span wave interaction, as reported in [19], [20] and [21]. It was found that short span WECs can interact with a larger extent of incoming wave crest than their span, increasing the accessible amount of wave power. These WECs were also found to be relatively insensitive to angular misalignment with the incoming wave crests.

\section{Objective and Motivation}

Utilizing all experimental and numerical findings gained so far, the main objective of this study was to determine if and how a WEC could be sized to achieve efficient wave energy conversion in an actual ocean wave climate, which featured waves of varying wave height and wave length throughout the course of a year. The main parameter by which success was quantified was the year averaged efficiency and thus energy capture of the WEC. The year averaged efficiency should not be confused with the efficiency at a given (optimal) design point which was typically much higher, but was rather indicative of annual total energy production which was what the operator of a WEC could sell on the power grid. The latter is one of two main factors in determining the levelized cost of energy, with the other being construction and operating cost of the WEC. A second important metric was the capacity factor, which was the ratio between average power produced and rated (generator) power. The capacity factor influenced the construction cost of the device, since it was a measure of how well the installed generation capacity was utilized. As the generator was expected to be a main portion of the construction cost, a larger capacity factor would indicate better use of this generator and thus a reduction in the cost of energy. A third metric was the power production time fraction, indicating during which fraction of the time the device would be delivering power to the power grid. Finally it was of interest to compare these key performance parameters of capacity factor and annual efficiency to other more established renewable electricity sources, e.g. wind power to determine if a WEC could be expected to become a competitive source of electric power production.

\section{Numerical Approach}

The numerical approach used in this work utilizes two models that had been developed and experimentally validated in past work. The potential flow model used an inviscid conservation of energy approach to estimate two dimensional wave cancellation efficiency. It was complemented by first principles viscous loss estimates to account for losses due to skin friction at the hydrofoils. This two dimensional potential flow model was complemented by a 
three dimensional radiation model that had been validated against observed radiation patterns in wave tank experiments, and allowed for quantitative estimation of the three dimensional radiation efficiency. Both models were based on conservation of energy principles and applied control volume analysis.

This section outlines the main WEC design parameters being investigated in the next section, followed by the numerical approach used to model the inviscid and viscous two dimensional flow features. The final section details the model representing three dimensional radiation and viscous drag effects encountered for a WEC with finite span.

\subsection{Wave energy converter geometry}

A two-dimensional sketch of a typical cycloidal WEC as considered in this paper, is shown in Fig. 1. In general it features one or more hydrofoils attached parallel to a horizontally oriented main shaft at a radius $R$, rotating clockwise at angular speed $\omega$, and submerged at a depth $y_{c}$, which is measured relative to a Cartesian coordinate system with $y=0$ being the undisturbed free surface. In this investigation, two hydrofoils were used based on previous results [13]. The orientation (pitch) of each hydrofoil was adjusted to produce the desired level of circulation $\Gamma$, which was kept constant in time. At any point on the free surface the vertical elevation was $\eta$ and peak-to-peak amplitude of the resulting wave field was $H$. The incoming ocean wave was assumed to travel in the positive $\mathrm{x}$ direction as shown in Fig. 1. The water depth $D$ was assumed infinite, i.e. deep water waves were investigated.

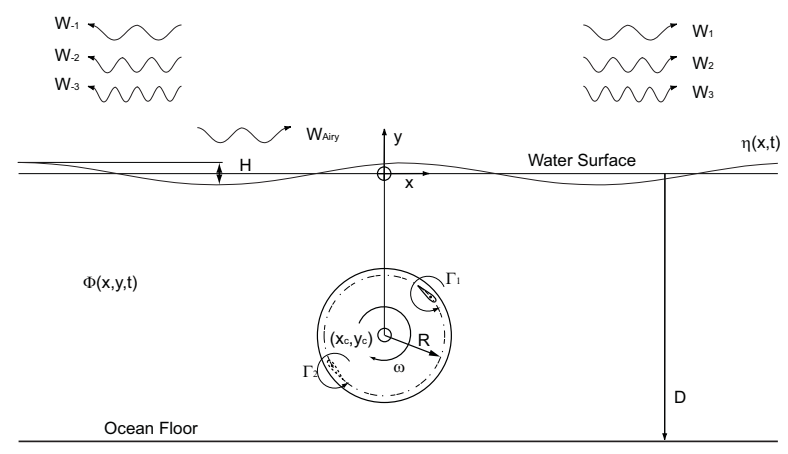

Figure 1: Cycloidal wave energy converter geometry

\subsection{Potential Flow Model}

Since a typical wave-induced flow field can be well described by potential flow theory, see for example Newman [22], a logical starting point to investigate the wave making and cancellation properties of a cycloidal WEC was to seek a potential flow solution. The process used in this investigation was outlined in Siegel et al.[13], where detailed derivation, validation, convergence study and comparison to results in literature were presented. A brief summary of this work follows here for completeness. The simplest representation of a two-dimensional hydrofoil correctly representing the flow induced in the far field was a point vortex of strength $\Gamma$ equal to the foil circulation. The complex potential for a vortex moving under a free surface with position $c(t)=x(t)+i y(t)$ in the complex plane has been developed in Wehausen and Laitone [23] to be,

$$
\begin{aligned}
F(z, t) & =\frac{\Gamma(t)}{2 \pi i} \ln \left(\frac{z-c(t)}{z-\bar{c}(t)}\right) \\
& +\frac{g}{\pi i} \int_{0}^{t} \int_{0}^{\infty} \frac{\Gamma(\tau)}{\sqrt{g k}} e^{-i k(z-\bar{c}(\tau))} \\
& \times \sin [\sqrt{g k}(t-\tau)] d k d \tau
\end{aligned}
$$

with $\Gamma(t)$ the circulation of the vortex, and $k$ the wave number. Eq. 1 satisfied both the kinematic and dynamic free surface boundary conditions at $y=0$. It is also important to note that in Eq. 1 the fluid was assumed to be infinitely deep. While the circulation $\Gamma(t)$ could be described in a time dependent fashion, this would constitute a numerical 
model of limited accuracy in terms of representing an unsteady hydrofoil of time varying angle of attack. This is because an actual hydrofoil would shed vorticity into its wake of an amount equal to the change in circulation, and this is not accounted for in Eq. 1. However, as has been shown in Siegel et al.[13], a constant circulation for each hydrofoil was most advantageous and thus implemented in the present work. This eliminated any shed vorticity in the wake. In the present work, the most efficient setup with two hydrofoils arranged opposite to each other, with opposite sign but equal magnitude circulation, was implemented.

Linear Airy wave theory was used to model the incoming wave. The velocity potential for a progressive linear deep ocean wave satisfying the linearized free surface boundary conditions was given in Newman [22] to be,

$$
\Phi_{\text {Airy }}(x, y, t)=\frac{H g}{2 \omega} e^{k y} \sin (k x-\omega t)
$$

where $H$ is the peak-to-peak wave amplitude, $g=9.81 \mathrm{~ms}^{-2}$ the gravity constant, $\omega$ is the wave frequency and $k$ is the wave number. Superposition was then utilized to determine the total velocity potential $\Phi_{\text {total }}=\Phi_{1}+\Phi_{2}+\Phi_{\text {Airy }}$, where $\Phi_{1}$ and $\Phi_{1}$ were the velocity potentials of each point vortex representing the hydrofoils. The positions of the two vortices were prescribed as a function of time. The coordinates for a vortex moving about a center of rotation $\left(0, y_{c}\right)$ with radius $R$ and frequency $\omega$ including a position offset $\theta$ relative to the incoming wave were,

$$
\begin{aligned}
& x(t)=R \cos (\omega t+\theta) \\
& y(t)=y_{c}-R \sin (\omega t+\theta) .
\end{aligned}
$$

Typically, the WEC created more than a single plain traveling wave. The wave height of each generated wave component could be determined by Fourier analysis. It was thus possible to determine the power associated with each wave $P_{\text {Airy }}$ by employing Airy wave theory which related wave power per unit length to wave height and period by:

$$
\begin{aligned}
P_{\text {Airy }} & =\frac{1}{8} \rho g H^{2} C_{g} \\
& =\frac{1}{32 \pi} \rho g^{2} H^{2} T
\end{aligned}
$$

where $\rho=1000 \mathrm{kgm}^{-3}$ was the density of water, $g=9.81 \mathrm{~ms}^{-2}$ the gravity constant, $C_{g}$ the wave group velocity and $H$ the Airy wave height. Since the wave power scaled linearly with the wave period $T$, higher harmonic waves of the same wave height contained less energy in proportion to their period. Also to be noted is a quadratic relationship between wave energy and wave height $H$. Based on wave power, the figure of merit for WEC design became the ratio of the power in the (desired) fundamental wave traveling in the positive $\mathrm{x}$ direction, $P_{1}$, compared to the power contained in all other harmonic waves,

$$
P_{1} / P_{\text {all }}=\frac{P_{1}}{\sum_{n=-\infty}^{\infty} P_{n}}
$$

since only the fundamental wave would interact with and cancel the incoming Airy wave, thus extracting power. The power ratio reached a value of one if only the desired down-wave traveling fundamental wave was created, and zero if no down-wave traveling fundamental wave was produced. The wave power analysis was based on energy conservation which was implicit in the unsteady Bernoulli equation, and a control volume analysis assuming that all energy leaving or entering at the up-wave and down-wave boundaries was contained in traveling Airy type waves. Thus, the power difference at both boundaries was to be provided or absorbed by the traveling point vortices. While the power ratio characterized the amount of power losses due to harmonic and up-wave traveling waves, also of importance was the amount of circulation required to generate a given fundamental wave height, which was defined as $H_{1} / \Gamma$. Since the fundamental wave height was linearly related to circulation as was shown in Siegel et al. [13], the amount of circulation required to cancel a wave of a given wave height could be calculated once $H_{1} / \Gamma$ had been determined for a wave period of interest. The losses due to harmonic and up-wave traveling waves in general will be referred to as harmonic losses in the following, defined as $P_{\text {harm }}=1-P_{1} / P_{\text {all }}$. 
While Eq. 4 was valid for harmonic waves as they were produced by the WEC in operation, the power $P_{i r r}$ in a typical irregular ocean sea state as recorded in the buoy data could be calculated by:

$$
P_{i r r}=\frac{1}{64 \pi} \rho g^{2} H_{s}^{2} T_{e}
$$

where $H_{s}$ was the significant wave height and $T_{e}$ the energy period of the sea state. Consequently, if the power of a harmonic wave was to match the power of an ocean sea state for a matching wave period, $H_{s}=\sqrt{2} H_{\text {Airy }}$. Due to findings reported by Fagley et al. [17], for the present parameter study harmonic wave cancellation simulations were used to estimate the efficiency for irregular wave buoy measured sea states, since it was found that the WEC could convert both types of waves with approximately equal efficiency if an appropriate feedback controller was employed.

\subsection{Viscous Loss Estimate}

In order to achieve the necessary wave height to cancel an incoming wave, the WEC blades needed to produce the required circulation $\Gamma$. For any hydrofoil, the circulation of the hydrofoil could be calculated as:

$$
\begin{aligned}
\Gamma & =\frac{c_{L}}{2} U_{f} c \\
c_{L} & =\frac{L}{1 / 2 \rho U_{f}^{2} c}
\end{aligned}
$$

with the hydrofoil chord $c$, non-dimensional lift coefficient $c_{L}$ and velocity $U_{f}$ of the foil, where $L$ was the lift force created by the foil per unit span. The lift coefficient $c_{L}$ along with the drag coefficient $c_{D}$ could be easily obtained from published data for a given hydrofoil shape, or calculated with panel codes that were available in the open source. Since there was excellent data available in open literature for the hydrofoil shape (a NACA0015) assumed in the present study, the results presented here relied on data from Sheldahl and Klimas [24] which were interpolated in pitch angle as well as Reynolds number. If due to the chosen WEC geometry and rotational period the required circulation could not be achieved before reaching the stall angle of attack, the circulation was limited to the circulation achieved just before hydrofoil stall. In this situation, wave termination would not be achieved but rather a reduced residual fundamental wave height $H_{1}$ would be found down-wave of the WEC.

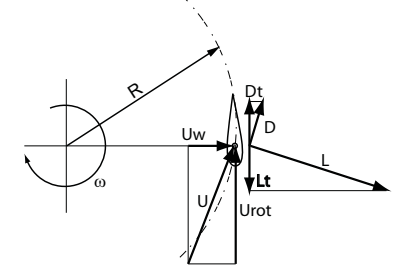

Figure 2: Hydrofoil Velocity and Force Vectors, from Siegel et al. [13]

The obtained lift and drag coefficients were used to calculate the shaft torque and thus power loss due to the tangential component of the drag force, $P_{d}=D_{t} R \omega$, Fig. 1 shows that this power is reducing the shaft power available. Using a conservation of energy approach, the power available at the shaft for two-dimensional flow $P_{S 2 D}$ of the WEC could be calculated as:

$$
P_{S 2 D}=\left(P_{\text {Airy }}-P_{1}\right)-P_{\text {harm }}-P_{d} .
$$

The power delivered to the shaft $P_{S 2 D}$ was thus for the scope of this paper defined as the portion of the wave power canceled by the WEC, minus the losses due to other (harmonic) waves generated, minus the losses due to viscous hydrofoil drag. This was a power model strictly based on two-dimensional assumptions and did not consider any three-dimensional fluid dynamic effects. It also ended at the main shaft without considering any bearing friction or generator and other electrical losses. It was possible to extend this model to a finite span hydrofoil as shown in the next section. 


\subsection{Three Dimensional Wave Radiation}

For a hydrofoil of finite span $S$, two additional effects needed to be considered. As the foil span became short compared to the wave length, three dimensional wave radiation effects needed to be considered as explored in Siegel et al. [20] and Siegel [21]. A brief summary of the radiation modeling approach and results follows for completeness, further details including validation of this model against experimental results as well as a resolution study can be found in the two references.

The surface elevation $\eta_{c}$ of a circular wave can be described as, $\eta_{c}(x, z, t)=H(r) \sin (\omega t-k r)$ with $\mathrm{x}$ and $\mathrm{z}$ the wave travel and wave normal coordinates, $\mathrm{k}$ the wave number, $r=\sqrt{\left(x-x_{0}\right)^{2}+\left(z-z_{0}\right)^{2}}$ the distance from the wave center, $\mathrm{T}$ the wave period and $\mathrm{t}$ time. If this wave is to conserve energy as it radiates outward, the wave height $\mathrm{H}$ has to decrease with distance from the wave generator. If the initial wave height is specified as $H_{0}$ at a finite distance $r_{0}$ from the center,

$$
H(r)=\sqrt{\frac{H_{0}^{2} r_{0}}{r}},
$$

the wave height does conserve wave energy resulting in a reduced wave height as the wave propagates away from the wave center at a $1 / r$ relationship. In Siegel et al. [20] it was shown that the wave radiated by a CycWEC could be represented with good accuracy by a series of circular waves arranged alonge the WEC span if these featured an azimuthal amplitude modulation of

$$
\begin{aligned}
H(r, \theta) & =\sqrt{\frac{H_{0}^{2} r_{0}}{r}} \cos \theta \\
\theta & =\arctan \left(\frac{z-z_{0}}{x-x_{0}}\right),
\end{aligned}
$$

where the angle $\theta$ was measured between wave travel direction and spatial location at the surface. For modeling the WEC, the waves generated could be approximated as a sum of $N$ individual semi-circular wave generators arranged along a line of finite length equal to the span $\mathrm{S}$ of the WEC blades, as expressed by

$$
\eta_{W E C}(x, z, t)=\sum_{n}^{N} \eta_{c n}, x>0 .
$$

In order to correctly represent the circulation distribution along the span of the hydrofoil, the heigth of these semi-circular waves was modulated in a elliptical fashion:

$$
H_{0}(z)=H_{c} \sqrt{\left(1-\left(\frac{z}{2 S}\right)^{2}\right.} .
$$

where $H_{c}$ was the wave height at the center of the WEC. Modeling the WEC generated waves using this approach and investigating their interaction with a straight crested incoming wave yielded wave patters that were consistent with those observed and measured in 1:10 scale wave tank experiments. A control volume analysis was then used to investigate the wave energy transport of all waves across the edges of a rectangular control volume enclosing the WEC. As energy was conserved in this model, analysis of the radiated waves allowed for determination of the power extracted by the WEC from the incoming wave.

The main result from this investigation was that a WEC of short span was able to interact with the incoming waves over a span wise extent that was larger than the actual span $S$ of the WEC and thus increased the amount of wave power accessible to the WEC. This added to the accessible incoming wave power $P_{\text {Airy }}$ by the amount $P_{\text {rad }}$. However, a larger circulation than for two-dimensional interaction was required to extract this wave power from the incoming wave, for details see Siegel et al. [20]. Wave radiation always increased the amount of power accessible to the WEC, although it might not have been possible to extract this power due to circulation limitations of the foils.

The second effect influencing the performance of a finite span hydrofoil was the induced drag of the foils due to end effects, which has been well explored in literature, see for example Glauert [25] and could be expressed by: 


$$
c_{d i}=\frac{c_{L}^{2}}{\pi e A R}
$$

where $c_{d i}$ is the induced drag coefficient, e the span efficiency factor which was assumed to be $e=0.9$ in this study, and AR the foil aspect ratio, $A R=S / c$. When the radiation power as well as the induced drag losses were added to the shaft power equation, the three-dimensional shaft power equation became:

$$
P_{S 3 D}=\left(P_{\text {Airy }}+P_{\text {rad }}-P_{1}\right)-P_{\text {harm }}-P_{d}-P_{d i} .
$$

where $P_{d i}=1 / 2 \rho U_{f}^{2} c_{d i} S c R \omega$ was the power loss due to the induced drag, which was in addition to the twodimensional foil drag power loss $P_{d}$.

The shaft power equation 15 included all fluid dynamic effects related to the interaction of the hydrofoils with the incoming waves. Clearly there were other sources of losses in this system which have been omitted in this model, on the fluid dynamic side these would be support structures and generator housing/nacelles which also interact with the wave and hydrofoil induced flow field. On the power conversion side, any generator or electrical losses beyond the shaft as well as bearing friction have been neglected. While these will be the subject of future research, it was felt that the present power model due to its simplicity allowed for good insight into the inherent trade offs and limits of wave energy conversion using the WEC system. A key result that could be derived from the shaft power was the device shaft efficiency, defined in this paper as the ratio between incoming wave power and shaft power:

$$
\eta_{s}=\frac{P_{s}}{P_{\text {Airy }}}
$$

The reference quantity of the incoming wave power $P_{\text {Airy }}$ for the 2D results was the specific wave power per $\mathrm{m}$ of wave crest, while for the $3 \mathrm{D}$ results it was the incoming wave power times the span of the hydrofoil.

\section{Results}

The following subsections introduce the wave climate data used as basis for the design of the WEC, followed by efficiency plots for different choices of radius, chord and span of the WEC. For a good design match the annual average efficiency as well as performance for different design choices with respect to generator power are presented in the following sections.

\subsection{Wave Climate}

The wave climate for which this initial design attempt of the WEC system was undertaken is that found off the North-East shore of the Hawaiian Island of Oahu, where buoy data was available online at CDIP [26]. The scatter diagram shown in the left portion of Fig. 3 has been adopted from the published source in that the actual hours per year for which a given sea state has been present are shown. The most dominant sea state can be seen to occur for a wave period of $T=8.5 \mathrm{~s}$ and significant wave height $H_{s}=1.75 \mathrm{~m}$.

However, it would be wrong to adopt this most likely sea state as a design point for a wave energy converter, if optimization of annual energy yield were the main objective. If the wave power of each sea state is taken into consideration, it can be seen in the right portion of Fig. 3 that the most energy was associated with a sea state of approximately $T=9.5 \mathrm{~s}$ and significant wave height $H_{s}=2.5 \mathrm{~m}$. The data plotted in the right portion of Fig. 3 was obtained by multiplying the annual hours of each sea state with the wave energy associated with that sea state calculated from Eq. 6. It therefore shows annual wave energy in units of $k W h / a / m$ of wave crest.

\subsection{WEC Efficiency}

With the overall efficiency being dependent on the canceled wave power as well as losses due to drag and losses due to harmonic waves as outlined in Eq. 9, it was of interest to investigate the dependency of these quantities on both wave height and wave period throughout the parameter range of the scatter diagram. Fig. 4 shows these quantities for a fixed wave period of $T=9 s$ as a function of wave height on the left, and as function of wave period for a fixed wave height $H_{s}=2.1 \mathrm{~m}$ on the right for one particular WEC design. 

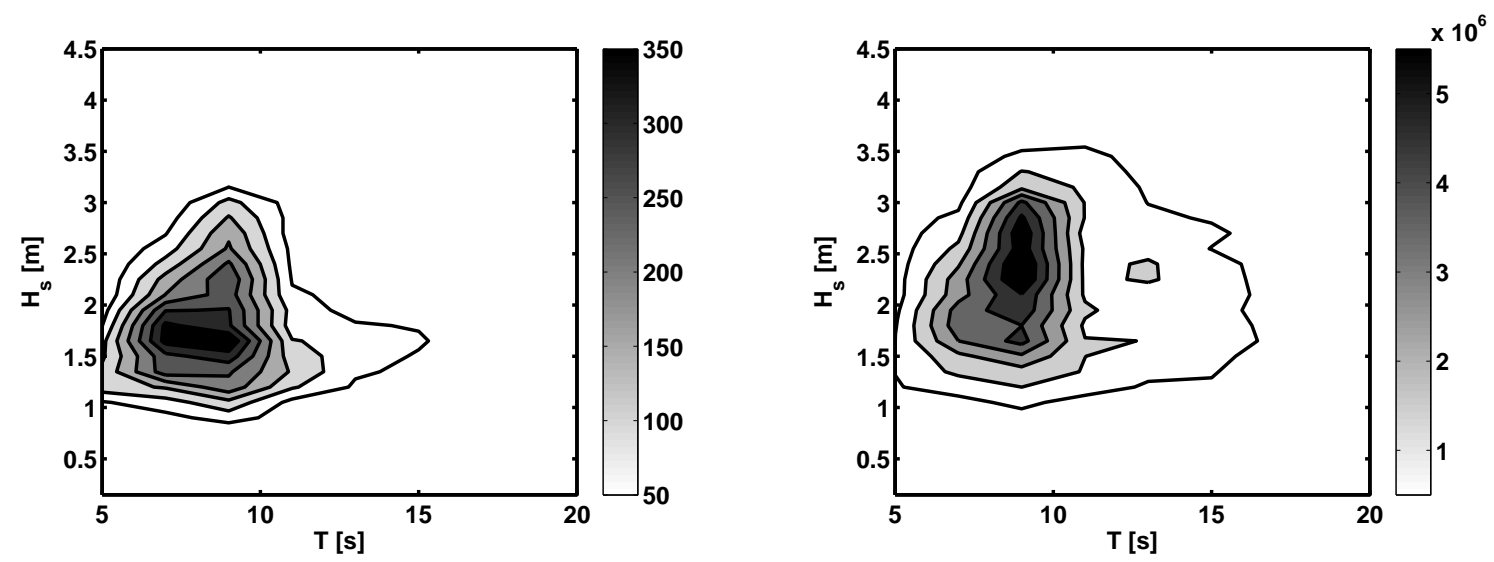

Figure 3: Probability distribution (left) and annual wave energy resource (right) at the Mokapu Point Buoy located off the North-East shore of Oahu, Hawaii for the year of 2010. The Probability is shown in units of [h/a], while the wave energy resource is shown in $[\mathrm{kWh} / \mathrm{a} / \mathrm{m}]$. Data from NOAA Buoy accessed through CDIP [26].
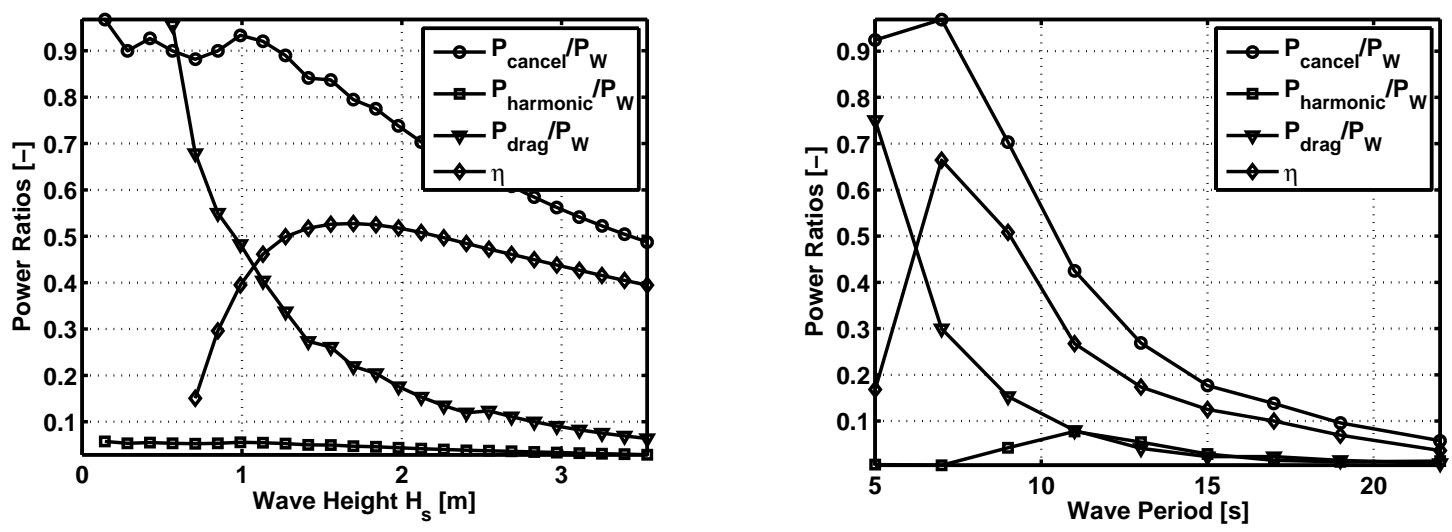

Figure 4: Canceled wave power, harmonic power losses, drag power and overall efficiency for a WEC with $R=5 m$ and $c=5 m$. Left, period $T=9 s$. Right, Wave height $H_{s}=2.1 \mathrm{~m}$. 
For a fixed wave period of $T=9 s$, the power $P_{\text {cancel }}$ that was extracted from the incoming wave remained approximately constant and at a high level of more than $90 \%$ up to $H_{s}=1 \mathrm{~m}$, and then decreased with increasing wave height. This decrease reflects the inability of the WEC to provide the necessary circulation required to cancel the entire incoming wave, and inspection of the lift coefficient (not shown) shows that the hydrofoil reaches its maximum lift coefficient at this point. The losses due to harmonic waves were small, at less than $5 \%$, and showed no strong dependency on wave height. Instead, the main source of losses were the viscous drag losses which were most dominant for small wave heights. In fact, for the parameters shown the viscous losses normalized by the incoming wave power, $P_{\text {drag }} / P_{w}$, exceeded the cancelled wave power for wave heights smaller than $H_{s}=0.6 m$. At this wave height and below the WEC would not be able to sustain its rotation any more and the resulting efficiency is negative, indicating that shaft power would have to be supplied in order to maintain rotation. This wave height will be referred to in the remainder of this paper as the cut-in wave height where the WEC will start producing shaft power. It is analogous to the cut-in wind speed of horizontal axis wind turbines, where a similar threshold wind-speed exists. The overall balance between cancelled wave power and power losses due to drag and harmonic waves leads to a maximum efficiency $\eta=0.52$, which was achieved at a wave height $H_{s}=1.75 \mathrm{~m}$ for the wave period and WEC design parameters shown. If conversely the wave height was kept constant while the wave period was being varied, as shown in Fig. 4 right, the extracted wave power can be seen to decrease with increasing wave period. This was due to two effects: As the wavelength increased with increasing wave period, the ratio between the the WEC diameter (which was fixed here) and the wavelength became smaller and smaller. This caused the ability of the WEC to cancel a given wave height to deteriorate, as the ratio between WEC diameter and wavelength moved further and further away from the optimal inviscid ratio of $2 R / \lambda=1 / \pi$ that had been established by Siegel [13]. In addition, the rotational speed of the hydrofoil decreased with increasing wave period, which led to a decrease in hydrofoil circulation. Both effects combined were responsible for the decreasing power extraction from the incoming waves seen in Fig. 4 right. The harmonic losses over the entire wave period range were again small, with a maximum of less that $10 \%$ of the incoming wave power. The drag losses however increased drastically for smaller wave periods. This was caused by two effects: As the wave period decreased, the available wave power decreased as well. In addition, the increased rotational speed of the hydrofoil caused larger drag losses. Both effects together were responsible for the steep increase of normalized drag power for smaller wave periods. Due to the tradeoff between extracted wave power and viscous as well as harmonic power losses an optimal efficiency existed, which was $\eta=0.68$ at a wave period of $T=7 \mathrm{~s}$. This will be referred to as the design point for a given wave height from here on, and is defined as the highest efficiency encountered.

Plotting the efficiency as a function of both wave period and wave height reflected the existence of an optimum combination of wave height and wave period where the WEC performed best. This can be seen in the four panels of Fig. 5, where there is a range of wave height and wave period combinations along which maximum WEC performance was achieved. As the design points varied with wave period and wave height, they formed a curved line along the peak in the efficiency contour plot.

The data presented in the four panels of Fig. 5 was obtained by keeping the device radius constant at $R=5 \mathrm{~m}$ and only varying the chord of the hydrofoil as indicated in the caption. Inspecting Fig. 5 left top reveals a distinct efficiency maximum. For the hydrofoil chord length of $c=1 \mathrm{~m}$ it was located at approximately a wave period of $T=7.5 \mathrm{~s}$ and significant wave height $H_{s}=1.5 \mathrm{~m}$ and reached about $30 \%$. For shorter wave periods the highest efficiency was found at larger wave heights, and vice versa. In a similar fashion, the cut-in wave height depended on the wave period as well, with larger cut-in wave heights at smaller wave periods, and smaller cut-in wave heights at larger wave periods. Both of these effects were caused by the trade-off between wave cancellation capability and visous losses as detailed in the discussion of Fig. 4 above.

The underlying physics causing this distribution for a given wave period (e.g. $T=7.5 \mathrm{~s}$ ) was related to viscous losses as well as hydrofoil maximum lift. Below the cut-in wave height, the viscous losses were larger than the power extracted from the wave leading to a negative efficiency or energy loss. This would prevent rotation of the WEC unless power was applied at the shaft. As wave height increased, so did the amount of energy that could be recovered. As the viscous losses were almost constant, this led to an increase in efficiency. The increase in energy recovered required a respective increase in hydrofoil pitch angle. The maximum efficiency was achieved once the hydrofoil just about reached its stall angle. Beyond that wave height, only incomplete wave cancellation was achieved and thus the efficiency declined asymptotically, without reaching zero. Thus, although not distinguishable due to the selection of plotting contour levels, below the cut-in wave height the efficiency was negative, while above the maximum efficiency line the efficiency remained positive for all larger wave heights. Despite this efficiency decrease, the amount of shaft 

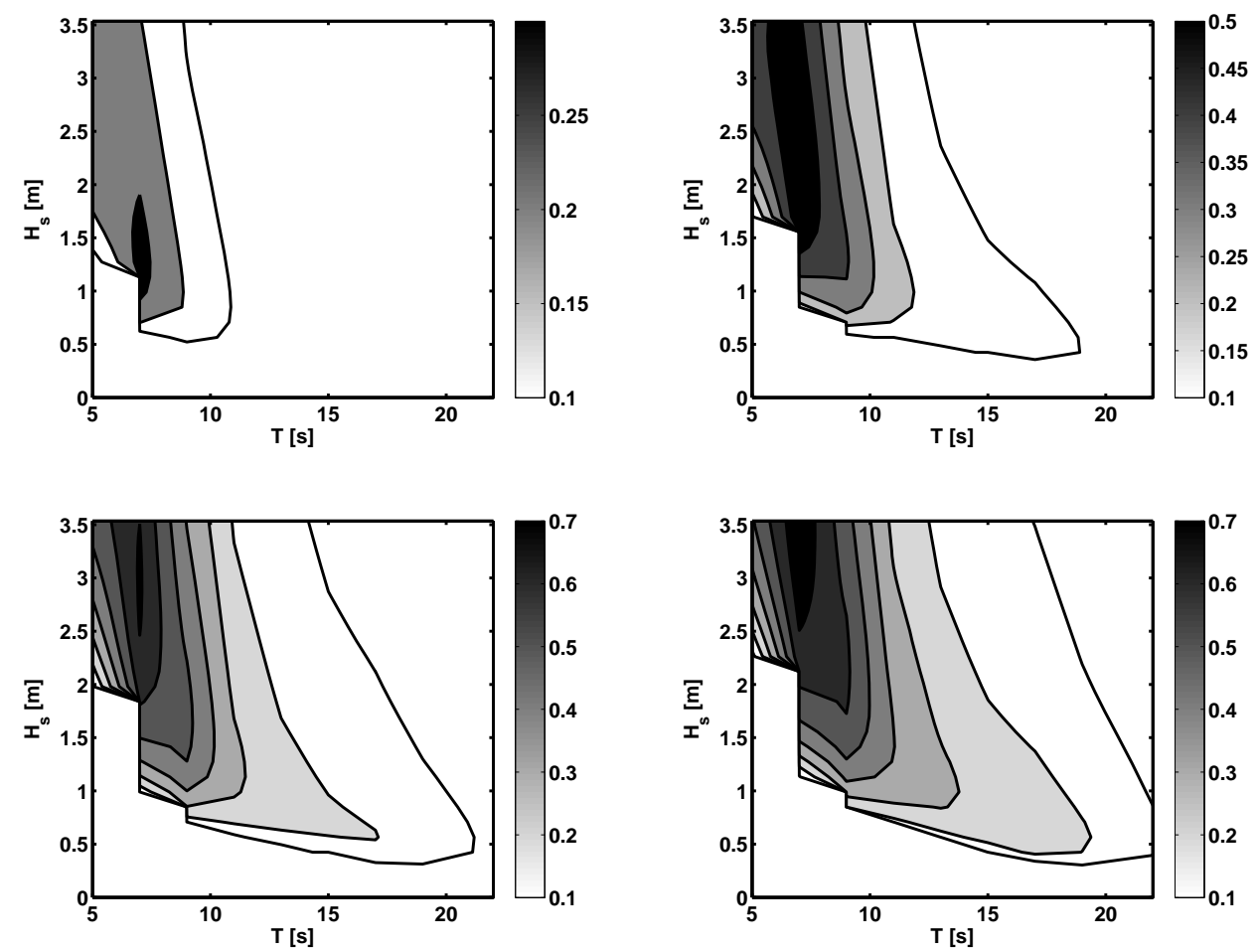

Figure 5: Impact of chord length on two dimensional WEC efficiency. The hydrofoil chord was varied from $c=1 \mathrm{~m}$ (top left) to $c=3 m$ (top right) to $c=5 m$ (bottom left) and $c=7.5 m$ (bottom right), while the WEC radius was kept constant at $R=5 \mathrm{~m}$
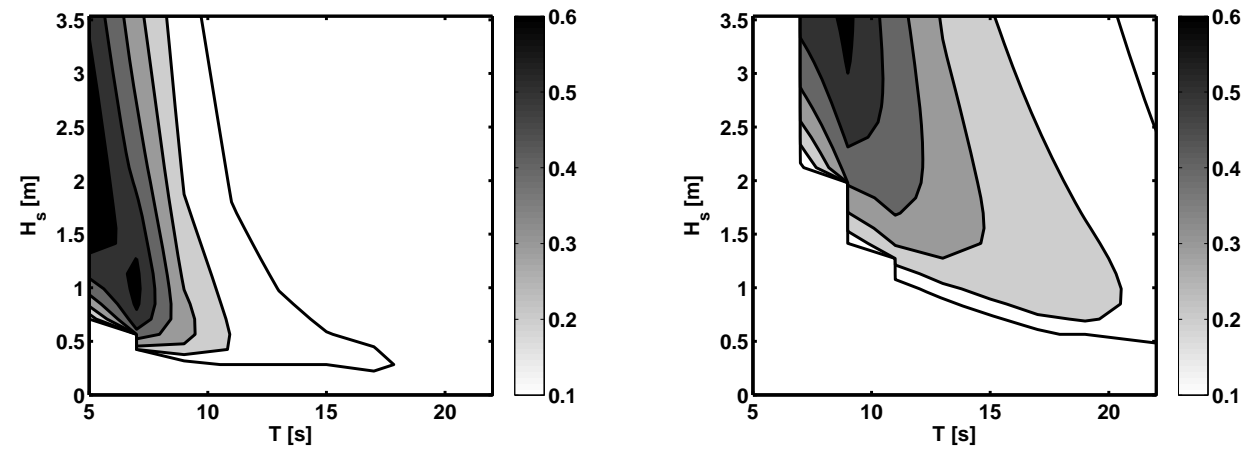

Figure 6: Impact of WEC radius on two dimensional WEC efficiency. The WEC radius was $R=3 m$ (left) and $R=7.5 m$ (right), with the same non-dimensional chord length of $c / R=1$ for both cases. 
power increased for larger wave heights as will be shown later.

As the wave period was varied, the cut-in wave height along with the maximum efficiency wave height changed. Towards smaller wave periods, a higher cut-in wave height was found. This was due to two effects: The incoming waves transported less and less wave power according to Eq. 6, and at the same time the increase in rotational speed of the WEC increased the viscous losses. Both effects together were responsible for the increase in cut-in wave height. Conversely, towards larger wave periods the viscous losses decreased and the wave power increased. However, at the same time the larger wave period hampered the ability of the hydrofoil to produce the necessary circulation due to the decreased rotational velocity, and thus limited the ability of the WEC to cancel the incoming wave. Therefore the efficiency of the WEC shown in the left top of Fig. 5 dropped below the lowest contour line of $10 \%$ at a wave period of $T=11 s$.

The qualitative behavior discussed above for design choices of radius $R=5 \mathrm{~m}$ and chord $c=1 \mathrm{~m}$ applied to all efficiency contour plots presented in this paper, along with many more not presented for brevity. However, the quantitative values for cut-in wave height and design wave height varied as radius and chord were changed. For changes in chord, the remaining panels in Fig. 5 show a shift of the design point to higher wave heights as the chord length was increased. This applied to all chord lengths up the maximum chord length of $C=7.5 \mathrm{~m}$ investigated. At the same time, the peak efficiency increased as well but only up to a chord length of $C=5 \mathrm{~m}$, beyond which it remained flat or decreased slightly. Thus, in terms of efficiency an optimal chord choice existed.

This behavior was consistent for other radius choices investigated, two of which are shown in Fig. 6. The nondimensional chord length $c / R$ was kept constant in both of these figures. Comparing the peak efficiencies achieved for both of these radii, it can be observed that neither the smaller nor the larger radius choice exceeded the performance of the $R=5 \mathrm{~m}$ design, indicating an optimum to exist for the choice of radius as well. However, the smaller radius achieved the optimal efficiency at a much lower wave period of $T=5 \mathrm{~s}$, while the larger radius design point occured at a larger wave period of $T=7 \mathrm{~s}$. Also, both the cut-in wave height as well as the design point wave height were increased for the larger radius compared to the smaller radius.

In summary, the WEC design parameters radius and chord both influenced overall wave to shaft energy conversion efficiency, as well as the wave height and period at which the design point with optimal efficiency was located. This allowed a WEC to be designed to optimally match a given wave climate, which is discussed in the following section.

\subsection{Matching of WEC Efficiency and Wave Climate}

While data in the previous section established the existence of an optimal design choice for both WEC radius and hydrofoil chord for a single wave height and period, the overall goal of WEC design was to match the performance of the WEC to the wave climate in which the device was designed to operate. Two different objectives can be set in this matching process. If the (positive) efficiency of the WEC covered the entire range of sea states shown in the left portion of Fig. 3, the WEC will produce shaft power at all times, leading to a power production time fraction of $100 \%$. This may be a desirable goal for a grid connected wave energy converter covering a base load on the power grid. It will not necessarily result in the highest annual power production, though, since in this case the efficiency maximum of the WEC will not be aligned with the most energy producing sea states shown in the right portion of Fig. 3. On the other hand, if the design goal was to maximize annual energy yield, the best possible match with annual wave energy and wave energy converter efficiency becomes the design goal. Since the wave period and height for both of these points are different, the two design objectives will lead to different wave energy converter geometries.

These two design options are graphically compared in Fig. 7. On the left side, a WEC was designed to cover the largest possible fraction of sea states occurring throughout the year. As a result, the $11 \%$ contour line almost entirely encloses the $10 \%$ scatter probability line of the wave climate data, which is shown as overlay. However, the peak efficiency of this WEC design is at a relatively small wave period of $6 \mathrm{~s}$, which is far away from the most energetic sea states in this climate, which are at about $9.5 \mathrm{~s}$ wave period. In order to optimize the annual energy yield, a WEC design is required that matches the peak efficiency of the WEC with the most energetic sea states. The WEC design in Fig. 7 right achieves a better (albeit not perfect) match, but at the cost of not capturing the lower wave period sea states due to higher cut-in wave heights at the lower wave periods.

In order to investigate these trade offs quantitatively, a parameter study with different WEC radii and chord lengths was conducted. Fig. 8 shows the two most important performance metrics, the average annual energy yield and the power production time fraction, for a number of different WEC radii and foil chord length combinations. The energy 

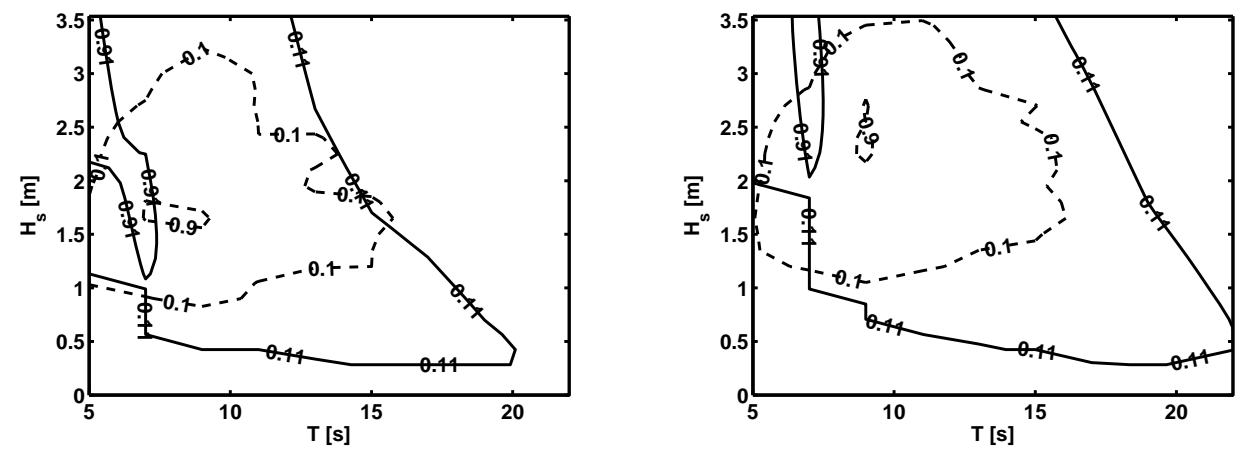

Figure 7: WEC efficiency contour lines at $11 \%$ and $91 \%$ of maximum efficiency (solid lines). A WEC chord of $c=4 m$ and WEC radius of $R=4 m$ (left) overlayed with scatter probability contour lines (dashed lines)is shown in comparison to a WEC chord of $c=5 m$ and WEC radius of $R=5 m$ (right) overlayed with annual wave energy contour lines (dashed lines)
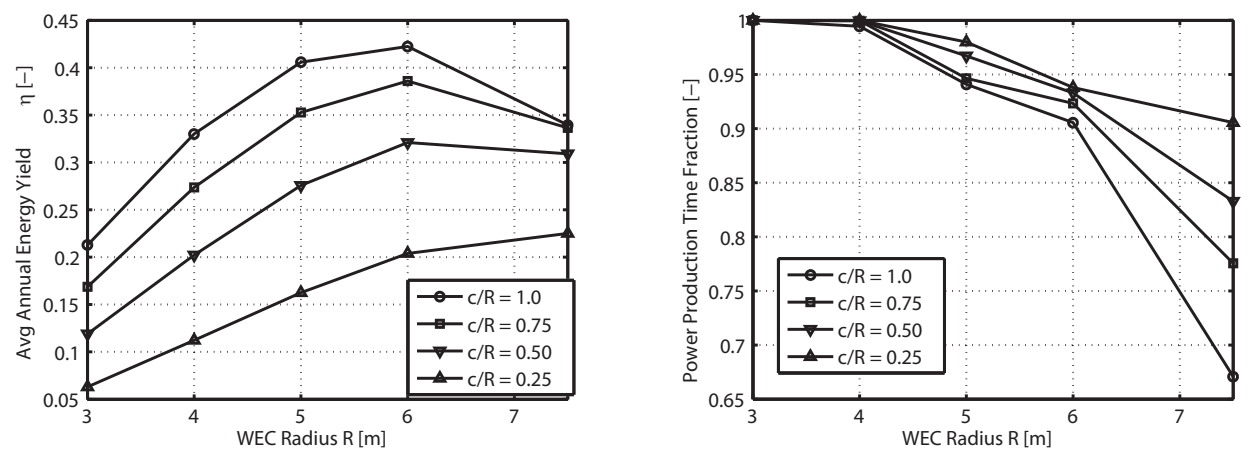

Figure 8: Average annual WEC efficiency as a function of WEC radius (left). Annual time fraction for power production (right). Data shown is based on infinite WEC span (two dimensional flow) and unlimited generator power, and specific for the Mokapu wave climate shown in Fig. 3.

yield features a distinct maximum for each non-dimensional chord length investigated, which shifted to larger radii for shorter chord lengths. This was caused by the ability to cancel the incoming wave, which required a certain amount of circulation. This circulation could be achieved by either a large chord and small radius, or vice versa. If chord and/or radius were too large, however, the cut-in wave height increased and thus the annual energy yield decreased, since wave energy at smaller wave periods and wave heights could not be converted to shaft power any more. The power production time fraction was however largest for the smallest WEC radius, and relatively independent of WEC chord length. This was due to the strong dependence of the cut-in wave height on the radius, where larger radii significantly increased the cut-in wave height.

The resulting annual energy yield, which was the product of device efficiency and wave resource is shown in Fig. 9. It was centered around the peak resource of $T=9.5 \mathrm{~s}$ and $H_{s}=2.0 \mathrm{~m}$ indicating a good match of device design and wave climate.

\subsection{Generator Capacity Limitation}

The device design to this point had been performed without consideration of generator capacity by assuming that any amount of shaft power could be handled by the generator and converted to electricity. This is evident in the shaft power plot shown in Fig. 10 left, which depicts the shaft power as a function of wave period and wave height. The maximum shaft power was encountered at a wave period of approximately $T=7 \mathrm{~s}$ and wave height of $H_{s}>3 m$, which 


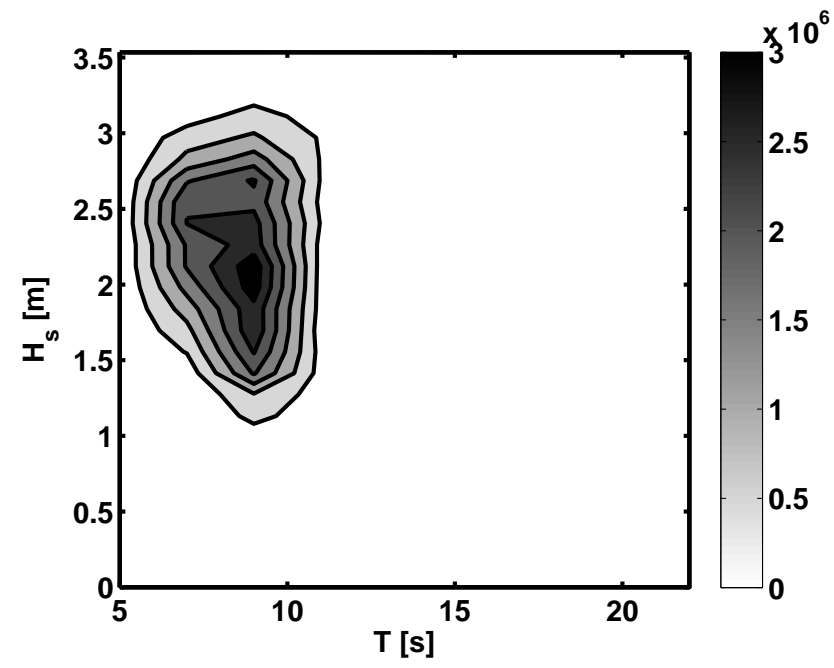

Figure 9: The annual energy yield at the WEC shaft for a WEC with $R=5 \mathrm{~m}$ and $c=5 \mathrm{~m}$. Units are $\mathrm{kWh} / \mathrm{a} / \mathrm{m}$.
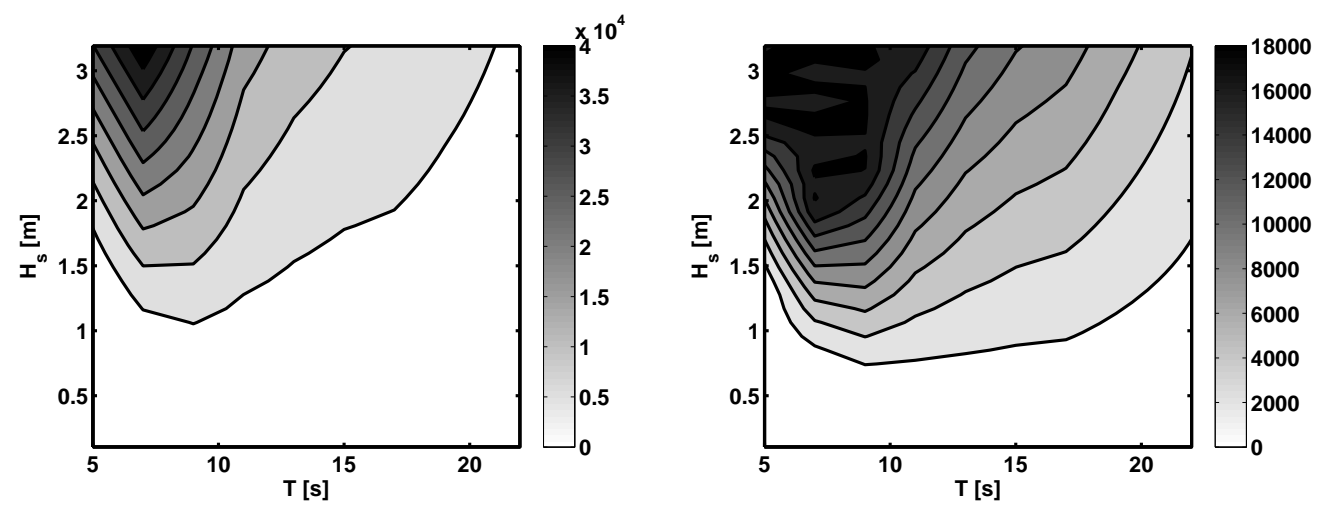

Figure 10: Shaft power in units of $W / m$ as a function of wave period and wave height for a WEC with $R=5 m$ and $c=5 \mathrm{~m}$. Power with no limitation on generator capacity (left) and a limit of $P_{\text {gen }}=18 \mathrm{~kW} / \mathrm{m}$ (right)

was well off from the maximum wave energy sea state shown in the scatter distribution. The actual values plotted also showed a shaft power beyond $40 \mathrm{~kW} / \mathrm{m}$ of wave crest, which was far larger than the average annual wave power in this climate of about $17 \mathrm{~kW} / \mathrm{m}$ of wave crest. A generator of $40 \mathrm{~kW} / \mathrm{m}$ would thus operate at a very small capacity factor, making the overall design uneconomical.

The design choice of a generator capacity of $18 \mathrm{~kW} / \mathrm{m}$ lead to the power output distribution shown in Fig. 10 right which was constant for a larger range of wave conditions, and resulted in full (rated) generator power output of $18 \mathrm{~kW} / \mathrm{m}$ during $20 \%$ of the time. However, some power was sacrificed in this process of reducing generator capacity since not all of the power available at some of the higher sea states with larger wave heights could be converted to electricity any more.

The overall trade off between capacity factor, annual energy yield and full time power fraction can be seen in the data shown in Fig. 11 top right, top left and bottom left, respectively, as a function of generator power. As generating power was increased, the annual energy yield was increased but levelled off beyond a certain generator power that was dependend on the WEC radius. However, this increase in energy production came at the price of a reduced capacity factor and full time power fraction. A likely design point for generator size might be chosen at about equal values for capacity factor and efficiency. For a NACA 0015 hydrofoil with a chord of $c=5 \mathrm{~m}$ and radius of $R=5 \mathrm{~m}$ this point was located at a maximum generator power of $P_{g}=18 \mathrm{~kW} / \mathrm{m}$ resulting in about $40 \%$ efficiency and capacity factor as 

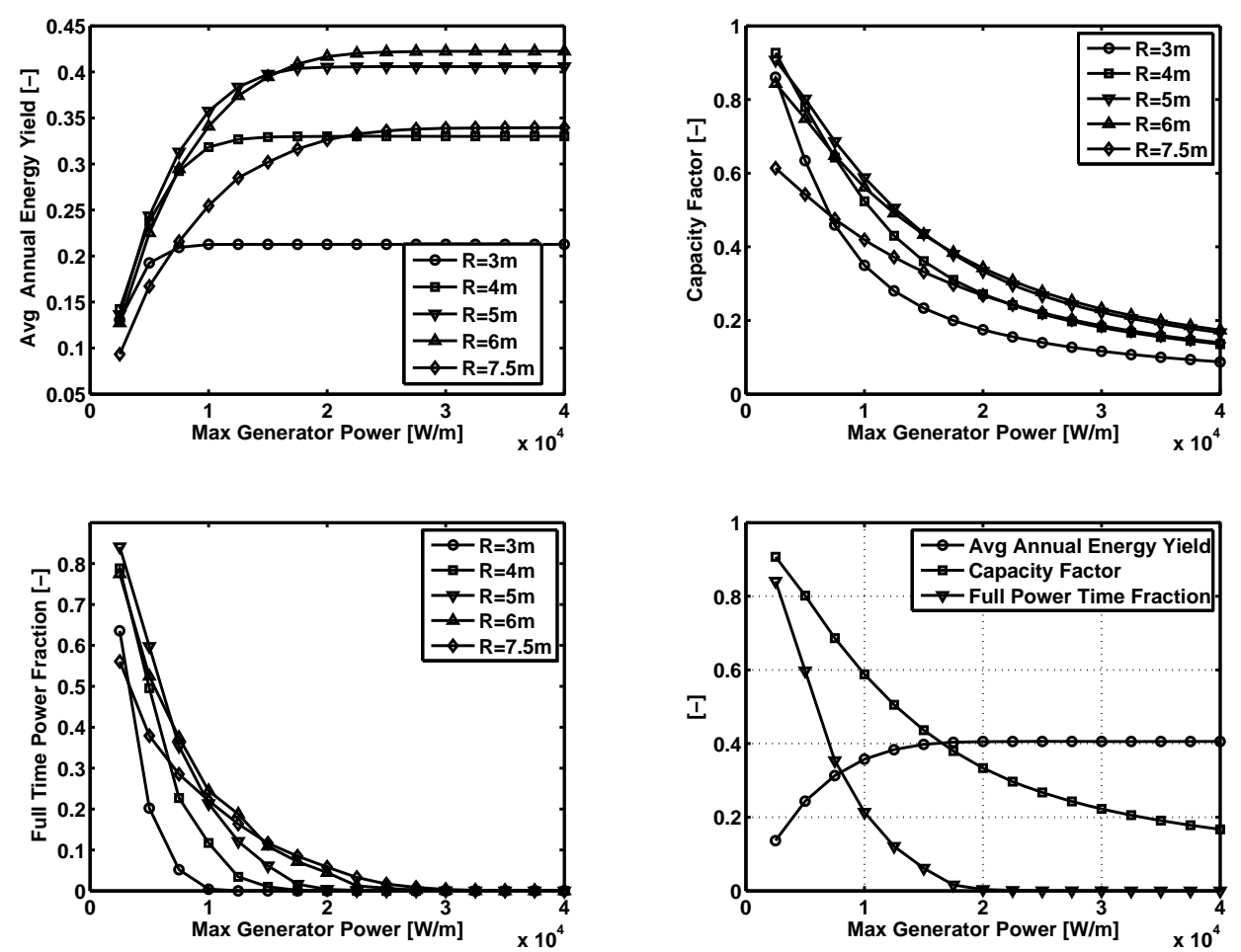

Figure 11: The average annual energy yield (top left), capacity factor (top right), and full time power fraction (bottom left) for WECs of varying radius as identified in the legend with a non-dimensional chord of $c / R=1$. Comparison plot of annual efficiency, capacity factor and full time power fraction for a WEC with $c=5 m$ and $R=5 m$ (bottom right). 


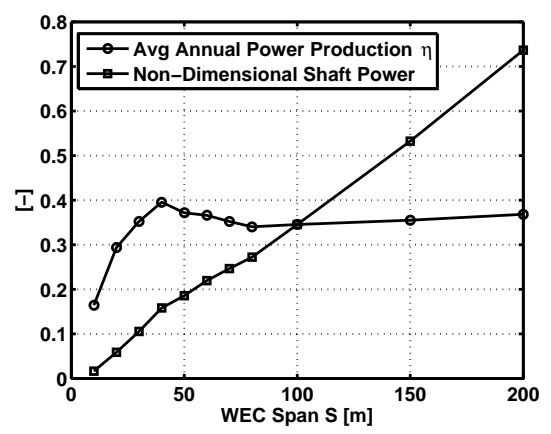

Figure 12: Annual average shaft power and annual efficiency as a function of WEC span. Three dimensional WEC radius $R=5 m$ and chord $c=5 m$. Data shown is based on unlimited generator power, and specific for the Mokapu wave climate shown in Fig. 3.

shown in the bottom right panel of Fig. 11.

\subsection{Three Dimensional Performance}

All results shown so far were made under the assumption of two-dimensional flow conditions, or an infinite span of the WEC hydrofoil blades. Since any actual WEC implementation will feature a finite span, it was of interest to investigate the impact of finite span on WEC performance. As was outlined in the setup section, two additional effects applied to a finite span WEC; an increased capability to cancel incoming waves over a spanwise extent exceeding the actual span of the WEC due to wave radiation, and an increased shaft power loss due to induced drag. Both effects become more dominant for shorter spans.

To explore the impact and trade off of both of these effects on the main WEC design parameters radius and foil chord length, the parameter study shown in Fig. 8 for an infinite span was repeated with a fixed finite span of $S=50 \mathrm{~m}$. The results of this finite span study (not shown for brevity) are qualitatively remarkably similar to the two dimensional result. In terms of quantity, the finite span results show a reduced optimal radius from $R=6 m$ to $R=5 m$ for the three-dimensional results, while the optimal non-dimensional chord choice remained the same at $c / R=1$ for both finite and infinite span WECs. Finite span effects also reduced the optimal average annual energy yield from $\eta=0.43$ in the two-dimensional case to $\eta=0.37$ in the three-dimensional case. These results indicated that the (performance reducing) induced drag effects outweighed any (performance increasing) wave radiation effects.

A parameter study was conducted to determine the optimal span for the chosen wave climate. The results shown in Fig. 12 left indicate an optimal efficiency of $40 \%$ for a foil span of $S=40 \mathrm{~m}$. For larger spans the efficiency was reduced and levelled off at about $35 \%$. However, the decrease in efficiency was more than compensated by the larger amount of incoming wave energy, thus the actual shaft power increased almost linearly with span for larger spans beyond the most efficient span of $S=40 \mathrm{~m}$. This allowed for larger power generation by increasing foil span in this wave climate.

The generator sizing results shown in Fig. 13 for the most efficient span of $S=40 m$ were qualitatively similar to the two-dimensional results shown in Fig. 11 bottom right. With a finite span, the actual generator power is now shown rather than the span specific power per $\mathrm{m}$ of span. The WEC with a radius of $R=5 \mathrm{~m}$ and chord of $c=5 \mathrm{~m}$ approached the maximum average annual power production of $40 \%$ for a generator power of $1 \mathrm{MW}$ or above. Any larger generator size did not yield any significant increase in power production. However, another design approach to optimize capacity factor and full time power production time over power production was also possible. In the extreme case, this would be a generator choice of $250 \mathrm{~kW}$, which would result in a capacity factor of $69 \%$ and a full time power fraction of $38 \%$. Such a design would be more advantageous in order to cover a base load on the power grid.

Only a detailed cost analysis including the operational considerations of the power grid operator will enable a decision on these design options. For example, if the WEC were connected to a large power grid that had only a small fraction of renewable power in its production mix, optimization of the annual power production would be desirable in order to maximize revenue generated from the WEC. On the other hand, if the WEC were operating on a very small 


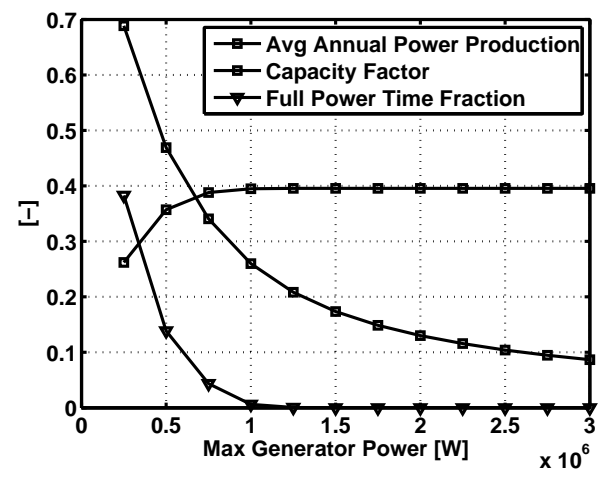

Figure 13: Generator sizing options for the most efficient WEC design for the Mokapu wave climate with consideration of three dimensional effects. WEC geometry $R=5 m, c=5 m$ and $S=40 m$.
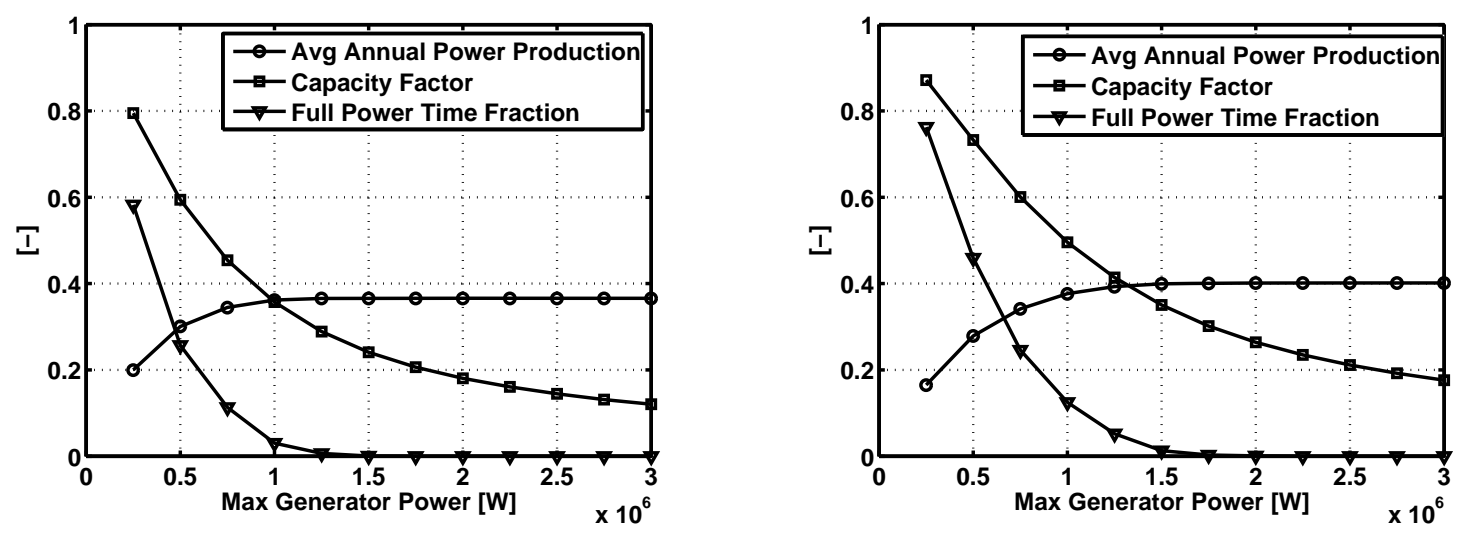

Figure 14: Impact of hydrofoil shape on WEC performance. Left, NACA 0015. Right, NACA63815.WEC geometry $R=5 m, c=5 m$ and $S=60 m$.

power grid where it was covering a large portion of the overall power production, continuous power delivery might be of higher importance and value than annual power production so that a base load on this grid could be covered in the best possible fashion. This situation might be typical for remote isolated locations like islands and other isolated power grids and communities with very expensive other means of (non-renewable) power generation.

For all results shown so far, a very basic, turbulent flow symmetric hydrofoil shape was used. The NACA 0015 hydrofoil is well established as a basic symmetric hydrofoil, but does not yield optimal performance in terms of lift to drag ratio or high lift generation capability. In order to demonstrate the impact of hydrofoil shape on WEC performance, a generic cambered hydrofoil of type NACA 63815 is shown in the right of Fig. 14 compared to the NACA 0015 performance on the left, with all other design parameters kept identical. The performance increase can be seen to be substantial for all choices of shaft generator power, and for all parameters of interest. While a detailed hydrofoil design optimization was beyond the scope of this study, Fig. 14 gives a glimpse at the performance improvement potential by optimization of hydrofoil shape.

Another approach to improving the WEC performance is to make the radius of the WEC adjustable. It has been shown in this paper so far that the radius has a profound impact on performance, since both the cut-in wave height as well as the efficiency depend strongly on it. At the same time simple mechanical arrangements are conceivable that enable implementation of an adjustable radius, while other design parameters like span or chord length of the hydrofoils cannot easily be changed during operation. The data shown in Fig. 15 left shows that the range of wave heights and periods for which power was produced has been extended to cover the entire range of sea states encountered. In 

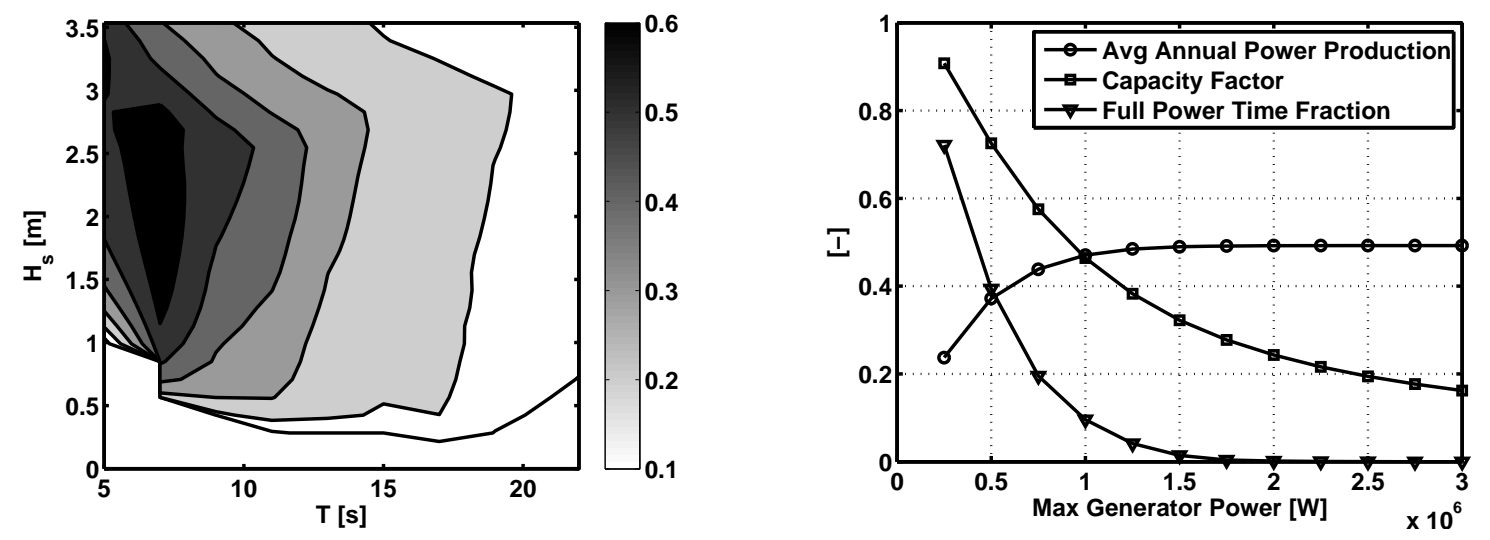

Figure 15: Impact of variable radius on WEC performance. Left, WEC efficiency. Right, generator sizing.WEC geometry variable radius of $R=3 \ldots 12.5 m, c=5 m$ and $S=60 m$; hydrofoil shape NACA63815.

comparison to the efficiency plots shown in Fig. 6, the variable radius covers an area of the plot that is a composite of both the smaller and larger radii shown in Fig. 6. This did not just enable power production for a larger range of sea states, but also improved the efficiency for most sea states compared to that of any of the fixed radius WEC designs. The main impact of this increase in conversion efficiency was an improvement of the average annual power production for all potential generator power choices over that of a fixed radius WEC. This can be seen by comparing the data shown in Fig. 14 right to that shown in Fig. 15 right, where no other design change has been made other than that of a fixed vs. adjustable radius. While the fixed WEC approached an average annual efficiency of $40 \%$ for large generator sizes, the variable WEC approached an efficiency of 50\% which is an improvement of more than $20 \%$ over the fixed radius design. However, only detailed cost and reliability analysis can show if this performance improvement would justify the added complexity and cost of a variable radius design.

\subsection{Discussion}

The linear potential flow investigations as reported by Siegel et al. [13] were advanced in the present work by an estimate of the gains and losses due to wave radiation, viscous drag and limits of hydrofoil performance due to hydrofoil stall. By investigating a range of sea states measured by a buoy, the annual energy yield and power production time fraction could be estimated for the first time for a chosen wave climate. The results of this investigation showed that an interdependence of the design parameters radius, foil chord length, foil span as well as generator power existed. Also, the optimal choice of these parameters was not just wave climate but also design objective dependent, where either annual power production or capacity factor and power production time fraction could be optimized at the cost of the other objective, respectively. Utilizing numerical results from potential flow solutions it was however possible to investigate the trade offs and arrive at an informed design decision.

Viscous losses were found to be the main factor governing the design, and the resulting optimal WEC radius was in all cases investigated much smaller than the inviscid optimal radius of $2 R / \lambda=1 / \pi$ that had been reported in Siegel et al. [13]. As the basic turbulent NACA 0015 hydrofoil shape employed for most part of this study does not provide a superior lift to drag ratio, utilizing a higher performance hydrofoil was investigated for one WEC geometry and found to improve WEC performance in all aspects significantly. Also, since a strong dependence of the overall efficiency on the non-dimensional radius $2 R / \lambda$ was apparent in the present investigation, it might make sense to employ a WEC of variable (adjustable) radius which would change the foil-to-shaft distance based on sea state. This type of device was shown to greatly improve overall annual efficiency for the wave climate investigated. This performance gain would however come at the cost of increased mechanical complexity of the WEC.

Even without adjustable radius, the present data indicated excellent annual power production and capacity factor in the relatively moderate wave climate of $17 \mathrm{~kW} / \mathrm{m}$ average wave power. It is worth while to put the results in perspective to more established renewable energy sources, with wind power being an appropriate choice as one of the 
most cost efficient renewable energy source. A overall efficiency including viscous losses at the design point of $70 \%$ was estimated for the WEC, which exceeds the inviscid theoretical limit of a wind turbine of 59\% and the typically achieved viscous efficiency of about $40 \%$ significantly. The results for the best power production optimized WEC geometry with $R=5 \mathrm{~m}, c=5 \mathrm{~m}$ and $S=60 \mathrm{~m}$ shown in Fig. 14 right achieved an annual average wave-to-shaft conversion efficiency of $40 \%$ and capacity factor of $42 \%$ with a maximum generator power output of $1.25 \mathrm{MW}$. This is beyond the performance of state of the art land based wind turbines with a capacity factor of $25 \%$, and approaches the best capacity factor of any offshore wind turbine reported at $46.7 \%$ to date [27].

While the present investigation was focused on a single wave climate, preliminary results for both weaker and stronger wave climates showed comparable results in terms of annual energy production efficiency and capacity factor. However, in stronger wave climates the optimal generator power was increased for a similar size WEC, improving the overall energy yield as one would expect. The results presented here should thus be considered typical, and similar or better results could be achieved in many locations world wide. However, the exact choice of the WEC geometry needed to be tailored to a specific wave climate for most efficient wave energy conversion.

One might argue that the optimal WEC design choice could be found by mathematical derivation rather than in the empirical / statistical fashion reported here. Since the WEC performance is described by Eq. 1 this may be true for the efficiency, albeit no closed solution of this equation has been reported in literature and thus possibly none may exist. However, the overall WEC design goal goes beyond determination of an optimally efficient WEC design for a single sea state, in that the WEC efficiency distribution needs to match a design wave climate as shown in this work. Since the latter is not available as a mathematical function, one has to resort to a semi-empirical engineering approach and/or iterative statistical means as were employed in the present study.

\section{Conclusions}

A lift based Cycloidal Wave Energy Converter (WEC) was investigated using potential flow numerical simulations in combination with viscous loss estimates based on published hydrofoil data. The impact of the main design parameters, namely the radius and hydrofoil chord of the WEC, were investigated in a parameter study conducted to match the performance of the WEC with the sea states of a typical ocean climate. As design target published scatter data from a buoy located off the shore of Oahu/Hawaii, USA was used. It was found that the performance of the WEC at the design point could exceed $70 \%$ wave-to-shaft conversion efficiency, but decreased as waves of different height and period were encountered. The efficiency distribution showed a marked cut-in wave height that was found to be a function of wave energy, and a drop-off of efficiency for larger wave heights as the circulation generation capacity of the hydrofoil was exceeded due to reaching its maximum angle of attack before stall. Both hydrofoil chord and WEC radius were interlinked in a non-linear fashion due to the quadratic relationship between flow velocity and lift generation of the hydrofoil.

With proper selection of the design parameters, a good match between hydrofoil efficiency and annual sea state distribution was achieved. When considering a finite power generation capability of the generator, a trade off between annual average efficiency and capacity factor of the WEC was observed. In order to optimize annual power production, a larger generator had to be chosen resulting in a less advantageous capacity factor, and vice versa. A reasonable design appeared to be selection of equal capacity factor and annual average efficiency, in which case the optimum design of the WEC in this particular wave climate yielded a value of $40 \%$ for both. This value was on par with published values for more established renewable energy sources like wind power.

\section{Acknowledgments}

The author would like to acknowledge the fruitful discussions with his colleagues at Atargis Energy, most importantly Dr. Seidel and Dr. Fagley. This material is partially based upon work supported by the Department of Energy under Award Number DE-EE0003635.

\section{Disclaimer}

This report was in part prepared as an account of work sponsored by an agency of the United States Government. Neither the United States Government nor any agency thereof, nor any of their employees, makes any warranty, 
express or implied, or assumes any legal liability or responsibility for the accuracy, completeness, or usefulness of any information, apparatus, product, or process disclosed, or represents that its use would not infringe privately owned rights. Reference herein to any specific commercial product, process, or service by trade name, trademark, manufacturer, or otherwise does not necessarily constitute or imply its endorsement, recommendation, or favoring by the United States Government or any agency thereof. The views and opinions of the author expressed herein do not necessarily state or reflect those of the United States Government or any agency thereof.

\section{References}

[1] Boyle G. Renewable Energy - Power for a sustainable future. Oxford University Press; 2004

[2] McCormick M. Ocean Wave Energy Conversion. John Wiley \& Sons; 1981.

[3] Mei CC. The Applied Dynamics of Ocean Surface Waves. John Wiley \& Sons, New York; 1983.

[4] Cruz J. Ocean wave energy: current status and future prepectives. Springer-Verlag; 2008.

[5] Wu TYT. Swimming of a waving plate 1961;10:321-344.

[6] Grue J, Mo A, Palm E. Propulsion of a foil moving in water waves 1988;186:393-417.

[7] Isshiki H. A theory of wave devouring propulsion (1st report). thrust generation by a linear wells turbine. J Soc Nav Arch Japan 1982;151(54).

[8] Isshiki H, Murakami M. A theory of wave devouring propulsion (3rd report). an experimental verification of thrust generation by a passivetype hydrofoil propulsor. J Soc Nav Arch Japan 1983;154(125).

[9] Isshiki H, Murakami M, Terao Y. Utilization of wave energy into propulsion of ships - wave devouring propulsion -. In: Naval Hydrodynamics. 1985,.

[10] Terao Y. Wave Devouring Propulsion System From Concept To Trans-Pacific Voyage. In: Proceedings of the 28th International Conference on Ocean, Offshore and Arctic Engineering. ASME; 2009,.

[11] Hermans AJ, van Sabben E, Pinkster J. A device to extract energy from water waves. Applied Ocean Research Computational Mechanics Publications 1990; Vol. 12, No. 4:5.

[12] Marburg C. Investigation on a Rotating Foil for Wave Energy Conversion. Master's thesis; TU Delft; 1994.

[13] Siegel SG, Jeans T, McLaughlin T. Deep ocean wave energy conversion using a cycloidal turbine. Applied Ocean Research April 2011; Volume 33 Issue 2:110-119. doi:http://dx.doi.org/10.1016/j.apor.2011.01.004.

[14] Siegel S, Jeans T, McLaughlin T. Deep ocean wave cancellation using a cycloidal turbine. In: 62nd Annual Meeting of the American Physical Society, Division of Fluid Dynamics, Minneapolis, MN. 2009,.

[15] Jeans T, Siegel SG, Fagley C, Seidel J. Irregular deep ocean wave energy conversion using a cycloidal wave energy converter. In: 9th European Wave and Tidal Energy Conference (EWTEC),Southampton, UK, September 5th 9th. 2011,.

[16] Siegel S, Fagley C, Roemer M, Mclaughlin T. Experimental investigation of irregular wave cancellation using a cycloidal wave energy converter. In: 31st International Conference on Ocean, Offshore and Arctic Engineering (OMAE). OMAE2012-83388; 2012,.

[17] Fagley C, Seidel J, Siegel S. Computational investigation of irregular wave cancelation using a cycloidal wave energy converter. In: 31 st International Conference on Ocean, Offshore and Arctic Engineering (OMAE). OMAE2012-83434; 2012,.

[18] Fagley C, Siegel S, Seidel J. Wave cancellation experiments using a 1:10 scale cycloidal wave energy converter. In: 1st Asian Wave and Tidal Energy Conference (AWTEC) Jeju Island, Korea, November 27-30. 2012,

[19] Fagley C, Siegel S, Seidel J. 3d efficiency analysis of cycloidal wave energy converters in oblique wave fields. In: 32nd International Conference on Ocean, Offshore and Arctic Engineering (OMAE). OMAE2013-10876; 2013,.

[20] Siegel SG, Fagley C, Seidel J. 3d wave diffraction efficiency of a double cycloidal wave energy converter. In: 10th European Wave and Tidal Energy Conference (EWTEC),Aalborg, DK, September 5th 9th. 2013,.

[21] Siegel SG. Wave climate scatter performance of a cycloidal wave energy converter. Applied Ocean Research 2014;submitted April:under review.

[22] Newman JN. Marine Hydrodynamics. MIT Press; 1977.

[23] Wehausen J, Laitone E. Surface Waves, Handbook of Physics, Vol.9. Springer-Verlag; 1960.

[24] Sheldahl R, Klimas P. Aerodynamic characteristics of seven symmetrical airfoil sections through 180-degree angle of attack for use in aerodynamic analysis of vertical axis wind turbines. Tech. Rep. SAND80-2114; Sandia National Laboratories; 1981.

[25] Glauert H. The elements of airfoil and airscrew theory. Cambridge University Press; 1947.

[26] CDIP. The coastal data information program. 2013. URL http://cdip.ucsd.edu/.

[27] Wikipedia . Capacity factor. 2014. URL en.wikipedia.org/wiki/Capacity_factor. 